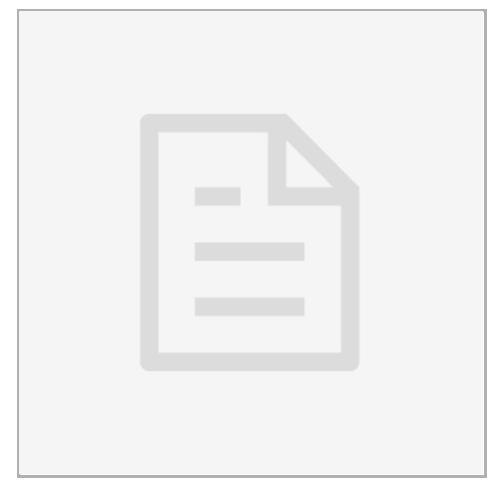

VERSION 2

JAN 17, 2019

\section{open $\boldsymbol{\text { Access }}$}

\section{DOI:}

dx.doi.org/10.17504/protocol s.io.w8ifhue

Protocol Citation: Lukas Snoek, H.steven Scholte, Tinka Beemsterboer 2019. Monthly Quality Control. protocols.io

https://dx.doi.org/10.17504/p rotocols.io.w8ifhue

License: This is an open access protocol distributed under the terms of the Creative Commons Attribution License, which permits unrestricted use, distribution, and reproduction in any medium, provided the original author and source are credited

Protocol status: Working We use this protocol and it's working

Created: Jan 17, 2019

Last Modified: Jan 17, 2019

PROTOCOL integer ID: 19434

\section{(3) Monthly Quality Control V.2}

\author{
Lukas H.steven
}

Snoek $^{1}, \quad$ Scholte $^{1}, \quad$ Tinka Beemsterboer $^{1}$

${ }^{1}$ University of Amsterdam / Spinoza Centre for Neuroimaging

Spinoza Centre, REC-L

Tinka Beemsterboer

\section{ABSTRACT}

The monthly quality control is a more extensive quality check of our 3T MRI system but also the other computers in the operator room.

The monthly quality control consists of the following components:

- Virus scan on scan-computer

- Moving eyetracker-files from DOS to Windows desktop

- Calibration of scanner (Philips protocol)

- 32 channel SNR test

\title{
Virus scan
}


Start a full virus scan of the MRI computer as follows:

- Right click virus scan icon in the bottom right corner; click "VirusScan Console"

- Select "Full scan", right click, and click "start"

You can do the calibration procedure during the virus scan.

\section{Delete eyetracker-files}

\section{Delete eyetracker-files ( min)}

The DOS-partition on the eyetracker computer is quite small, so once a month all edf-files (i.e., eyetracker-data files) should be deleted during the Monthly Quality control.

- Go to the 'filesystem' mode of the eyetracker-computer:

- start up the computer as usual

- click 'exit eyelink'; you'll boot into the 'filesystem' mode.

- delete all contents in the /data folder (all files should end with .edf).

\section{Calibration procedure, general}

\section{Calibration procedure ( 1.5 hours)}

Some general notes:

- The procedure leads you through a series of tests, which instructions are summarized below. The procedure is also documented on the scan-computer itself.

- All tests (in the following steps) need to be passed; if one or more tests failed, let Lukas know by mail. Data/results from the tests do not need to be saved

- All tests are performed without a headcoil; unplug and remove from bore.

- For most of the tests, a bottle or phantom (orb filled with liquid) is used. Check before testing whether the bottle is filled completely (there should be no -- or just a small, not more than 2 $\mathrm{cm}$ in width -- bubble present); if not, let Lukas know (you can go ahead with the protocol).

- All bottles are outlined user the laser on their center.

- After starting the adjustment/scan, you might hear/see the table moving; this is to disengage the posterior coil, which is (often) necessary during the recalibration procedure.

- Sometimes, the scan-computer might indicate that the phantom/bottle position needs to be adjusted; mess around with its initial position (i.e., move to the right/left/front/back) on the table until it works (this might take a while and can be annoying...).

To start the calibration procedure ...

- Navigate to "Start Service Application" (start menu $\rightarrow$ Service Application).

- You'll get a pop-up prompting to fill in our username (use "Lukas Snoek") and the reason for service (use "Planned Maintenance")

Clicking the folder will reveal several adjustments/scans. In the following recalibration procedure, 
we will execute only a subset of these adjustments.

- Now, go to the "Installation" tab. On the left side, you'll see different types of "procedures". We'll only do some System Level procedures.

\section{General instruction}

For the calibration procedure, run the tests below in the indicated order. After each test, the result ("Passed" or "Not passed") will be displayed in the upper-left corner of the window. In case a test didn't pass, send an email to Lukas about which test failed.

If you get the error: "Currently Field Service Login is not allowed. Ensure the system is activated.", just log out of the scan computer and try again.

\section{Calibration procedure}

\section{$5 \quad$ F0 determination}

- Bottle and position: 3 liter bottle, horizontally placed, lid towards bore

- Click "next", "next", and once the frequency appears click "Stop". Check the results.

\section{Multitransmit RF calibration}

- Bottle and position: 1 liter bottle, horizontally placed on holder, lid towards bore

- Follow instructions on screen

$7 \quad$ RF power ref. cal. Body coil

- Bottle and position: 3 liter bottle, horizontally placed, lid towards bore

- Follow instructions on screen

$8 \quad$ Pickup coil tripl. cal. Body Coil

- Bottle and position: 3 liter bottle, horizontally placed, lid towards bore

- Follow instructions on screen

\section{$9 \quad$ MR Eddy Current \& osc cal (std) ( 15 min.)}

- Bottle and position:

- Blue spherical ECC-phantom on own placeholder

- Outline as usual

- Most likely it will indicate the phantom-position is off; adjust along the instructions on screen (e.g. Z-direction: -2 = $2 \mathrm{~cm}$ further into bore) 
- Follow instructions on the screen.

\section{MR Eddy Current \& osc cal (par) ( 15 min.)}

- Like previous step.

- Blue spherical ECC-phantom on own placeholder

- Outline as usual;

- Follow instructions on the screen.

11 Channel delay and FID shimming

- Bottle and position: 3 liter bottle placed on top of two foam pads (see below)

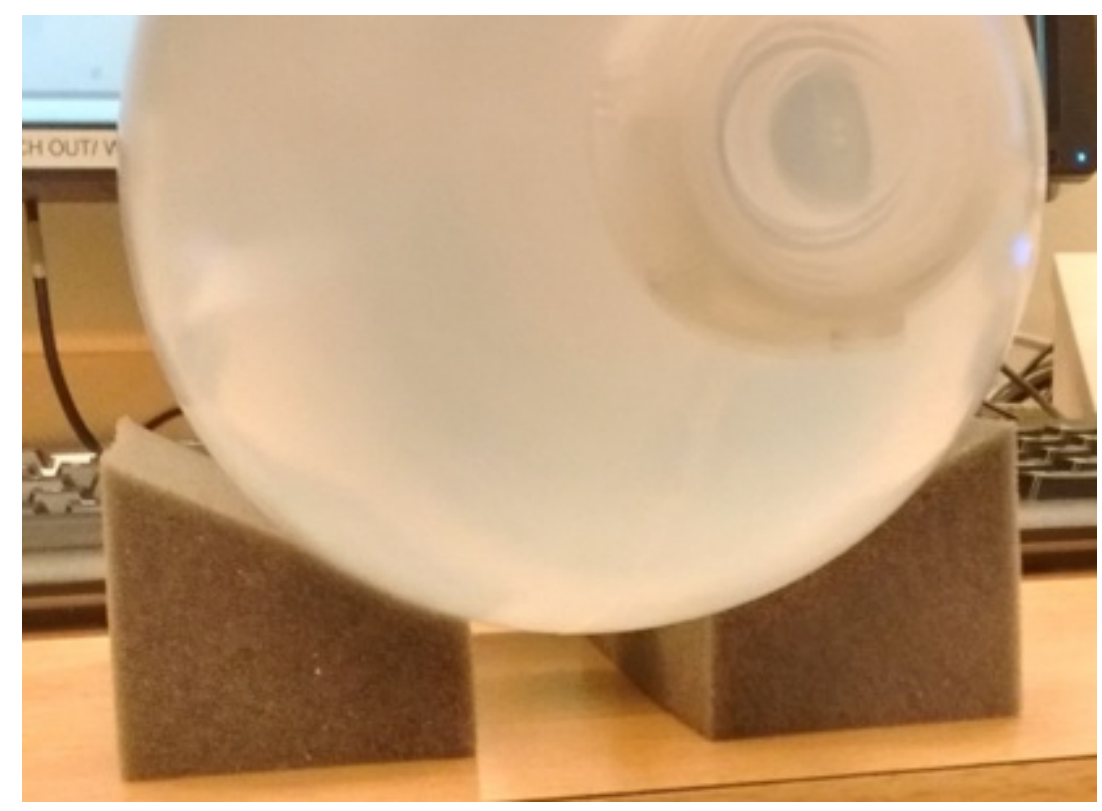

12 ECC analysis

- Bottle and position: 3 liter bottle, vertically placed

- A new window pops up (if not, alt+tab until "STT application" is visible); click "proceed"

- You probably have to re-align the bottle (see instructions on screen)

- Once correctly aligned, the test will start (takes about 8 minutes)

\section{SHIM-PS calibration ( 16 min.)}

- Bottle and position: 3 liter bottle, vertically placed

14 Grad. fine gain cal. (4x)

- No bottle (!), but using the PIQT-headcoil and phantom, yet with the phantom 'lying down' (horizontal) instead of 'upright' (vertical, as is normal for the regular PIQT-scan) for the FIRST TWO scans (std-cor, par-cor).

- Note the orientation in the image below; the 'edge' on the left side of the phantom (looking 
towards the bore) should fit exactly in the left side of the holder; the right side doesn't "fit perfectly" (it's slanted). Don't worry; if it's not placed correctly, the scan will stop automatically (after half a minute or so)

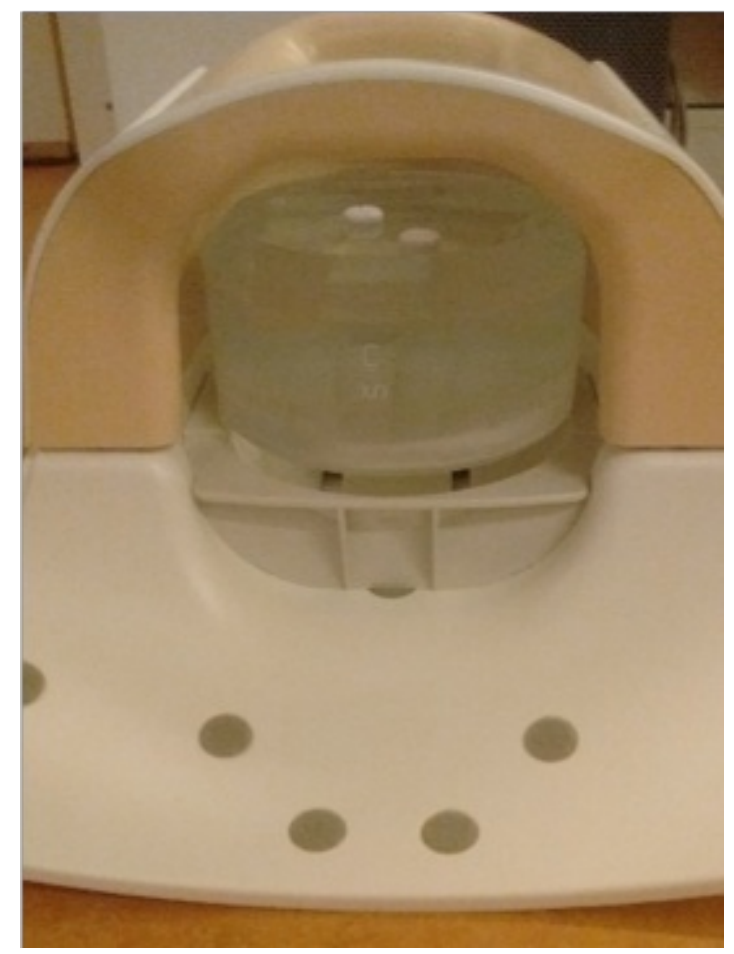

\section{2 channel SNR test}

\section{2 channel SNR test ( 30 minutes)}

This scan tests whether the 32-channel headcoil functions properly.

To start the protocol, do the following:

- Go to: System $\rightarrow$ STP (System Performance Tool),

- expand the Batch-files folder,

- and within the IQT folder select the SENSE-HEAD-32 folder,

- right-click the batch-file, and press "Run Batch".

- Follow the instructions on screen relating to phantom positioning and once everything is positioned correctly, click "proceed"; the scan starts automatically.

If you get an error about "Scan definition failed", restart the batch interpreter and start the SENSE-HEAD-32 scan again.

- After the scan is finished, you'll get a pop-up with information/specs of the just-executed scan; ignore this.

- Then, go to ImageView, 
- select the scan (SENSE_HEAD...) and click Tools $\rightarrow$ Generate Reports

- This opens a window in which specs for all the 32 channels of the headcoil.

- The number of the channel is indicated under the header "Scan_name" (e.g. 'SH32H_1', 'SH32H_2'); here, the number after the underscore indicates the channel $\mathrm{nr}$ (i.e. 'SH32H_21' is channel 21).

Note: in the HTML-report, there is no channel 23, but this is expected (problem with this particular SPT test) - so this is not an issue.

- In the QC excel file - under "maandelijks", write down the SNR (which is indicated by the header 'S/N (B)') for each channel.

Note

If any of the values in the HTML-window are marked red, let Lukas know!

\section{Misc.}

16 Clean the copper strips from the door to the scanner with some alcohol (only has to be done once every two months).

Do this in the following months:

- February

- April

- June

- August

- October

- December 